DOI: 10.12731/wsd-2017-4-2-74-90

УДК 616.12 - 008.331

\title{
ПРИМЕНЕНИЕ МЕТОДОВ МАТЕМАТИЧЕСКОГО МОДЕЛИРОВАНИЯ В ПРОГНОЗЕ РАЗВИТИЯ НЕБЛАГОПРИЯТНОГО ТЕЧЕНИЯ АРТЕРИАЛЬНОЙ ГИПЕРТОНИИ У ЖЕНЩИН
}

Яскевич Р.А., Москаленко О.Л.

Цель. Изучение возможности применения методов математического моделирования для прогноза клинического течения артериальной гипертонии у женщин.

Материалы и методы. Обследовано 84 женщиины в возрасте 20-60 лет (средний возраст 45,3 лет). Обследование включало клинические, инструментальные и лабораторные методы исследования. В качестве математической основы использовалась методика структуризачии и анализа разнотипных статистических данных в условиях непараметрической неопределенности.

Результаты. В ходе проведенного исследования по результатам математического моделирования, с применением методики распознавания образов, был сформирован индивидуальный набор признаков (факторов риска) из перечня показателей, обуславливающих риск развития прогнозируемого состояния (осложненное течение АГ), что позволило построить прогнозные номограммы, на которых выделены области низкого, среднего и высокого риска неблагоприятного течения АГ у женщин, что не только позволит рассчитать степень риска, но и определить параметры необходимого изменения уровня управляемых факторов риска, определяюших нахождение в зоне высокого риска, и, воздействуя на них проводить профилактические мероприятия. Установлено, что на клиническое течение АГ у женшин оказывает влияние повышение показателей инсулинемии, тощаковой и постпрандиальной гликемии, ИМТ, ОХС, уровня АД, то есть, симтомокомплекс метаболического синдрома.

Заключение. Использование метода реструктуризации и анализа разнотипных статистических данных в условиях непараметрической неопределенности позволяет прогнозировать и оценить тяжесть кли- 
нического течения АГ у женщин. Наиболее значимыми факторами, влияюшими на тяжесть клинического течения АГ у мужчин, являются показатели инсулинемии, гликемии, ИМТ, ОХС, уровни АД.

Ключевые слова: артериальная гипертония; прогноз; математическое моделирование.

\title{
APPLICATION OF METHODS OF MATHEMATICAL MODELING IN THE FORECAST OF DEVELOPMENT OF ADVERSE CURRENT OF ARTERIAL HYPERTENSION IN WOMEN
}

\author{
Yaskevich R.A., Moskalenko O.L.
}

The purpose of the study. Studying the possibility of using mathematical modeling methods for predicting the clinical course of arterial hypertension in women.

Materials and methods. 84 women aged 20-60 years (mean age 45,3 years) were examined. The survey included clinical, instrumental and laboratory methods of investigation. As a mathematical basis, we used a technique for structuring and analyzing heterogeneous statistical data under conditions of nonparametric uncertainty.

Results. In the course of the conducted research on the results of mathematical modeling, using the pattern recognition technique, an individual set of signs (risk factors) was formed from the list of indicators that predetermined the risk of development of the predicted state (complicated course of hypertension), which made it possible to construct forecast nomograms, medium and high risk of adverse course of AH in women, which will not only allow us to calculate the degree of risk, but also to determine the parameters of the required change of the level of managed risk factors that determine the presence in a high-risk zone, and, by influencing them to carry out preventive measures. It was found that the clinical course of hypertension in women is influenced by an increase in insulinemia, fasting and postprandial glycemia, BMI, OXC, and blood pressure, that is, a simtomocomplex of the metabolic syndrome.

The conclusion. The use of the method of restructuring and analysis of heterogeneous statistical data in conditions of non-parametric uncertainty makes it possible to predict and evaluate the severity of the clinical course of AH in women. The most significant factors affecting the severity of the clinical course 
of hypertension in men are the indicators of insulinemia, glycemia, BMI, OXC, blood pressure levels.

Keywords: arterial hypertension; predict; mathematical modeling.

Введение: Несмотря на некоторый прогресс в профилактике и лечении хронических неинфекционных заболеваний (ХНИЗ), болезни системы кровообращения (БСК) остаются ведущей причиной заболеваемости, инвалидности и смертности во многих странах мира, в том числе и в России $[13$, с. $55-57 ; 14$, с. $328 ; 20$, с. $94-96 ; 21$, с. эл. ресурс; 27 , с. 138]. В формировании стратегии профилактики сердечно-сосудистых заболеваний (СС3) изучение особенностей распространенности и выраженности факторов риска (ФР) ССЗ среди населения является одним из ключевых вопросов. Вместе с тем необходимо учитывать региональные особенности при разработке эффективных систем скрининга и контроля факторов риска среди населения различных регионов страны и в том числе среди жителей Сибири и Крайнего Севера $[5$, с. $23-25$; 13 , с. 55-57; 14, с. 328; 20, с. 94-96]. А разработка и внедрение в клиническую практику эффективных средств их первичной и вторичной профилактики, в том числе с использованием средств вычислительной техники, позволит эффективно воздействовать на эти факторы и снизить риск развития самого заболевания или риск его неблагоприятного течения $[9$, с. $18 ; 16$, с. 393-407; 24, c. $79-81 ; 26$, c. $672-677 ; 29$, c. $157-162]$.

Вопросам разработки прогноза развития различных заболеваний и патологических состояний за последнее время посвящено множество исследований [3, с. 19; 4, с. $158-167 ; 17$, с. эл.ресурс; 18; эл.ресурс], в том числе и прогнозу развития и клинического течения СС3 [22, 23, 25, 28]. Как правило в большинстве случаев используются линейные, статические математические прогнозные модели, основанные на подсчете баллов или прогностических индексов, представляющих сумму условных цифровых оценок признаков, влияющих на прогноз. Однако в плане информативности и повышения точности прогноза, предпочтительней построение динамических рядов признаков, полученных в различные временные периоды [6, с. 382; 7, с. 174; 8, с. 270].

Лапко А.В. с соавт, (1996 г.) для разработки прогноза развития нарушений углеводного обмена у коренных жителей Севера в качестве математической основы применили непараметрические алгоритмы распознавания образов [8]. Поликарпов Л.С. с соавт., (2005 г.) также применили непараметрические алгоритмы распознавания образов для создания системы 
прогноза метеотропных реакций у больных гипертонической болезнью (ГБ) [12]. Графическая интерпретация критериев прогноза уровней осложнений СС3 по значениям климатических факторов была представлена в виде номограмм. Каждая номограмма соответствовала определенной форме осложнений, где были выделены зоны низкого и высокого риска.

В экологических условиях Севера и Сибири осложнение заболеваний сердечно-сосудистой системы в значительной степени определяется действием экстремальных климатических факторов. Однако задача количественного прогноза метеотропных осложнений заболеваний сердечно-сосудистой системы для районов Севера и Сибири до сих пор не решена, что и определяет медико-социальную значимость этой проблемы и обуславливает потребность в её изучении.

Цель исследования: Изучение возможности применения непараметрических алгоритмов распознавания образов для прогноза развития неблагоприятного течения (с высоким риском развития ИБС и мозгового инсульта) артериальной гипертонии у женщин.

Материалы и методы: В обследовании приняли участие 84 женщины, г. Красноярска в возрасте 20-60 лет (средний возраст 45,33 [44,19-46,47] лет). В группе обследованных лиц с артериальной гипертонией было 54 чел. (средний возраст 46,91 [45,46-48,35] лет), а в группе контроля - 30 чел. (средний возраст 42,50 [41,07-43,93] лет) с нормальными уровнями АД.

Клиническое обследование больных включало врачебный осмотр, анкетирование, двукратное измерение артериального давления, эхокардиографию, электрокардиографию, суточное мониторирование ЭКГ. Лабораторное обследование включало определение уровней липидов крови, иммунореактивного инсулина (ИРИ), проведение перорального глюкозотолерантного теста (ПГТТ).

В качестве математической основы выбрана методика структуризации и анализа разнотипных статистических данных в условиях непараметрической неопределенности [6, с. $382 ; 7$, с. $174 ; 8$, с. 270]. В отличие от известных аналогов преимущество предлагаемых алгоритмов классификации объясняется их математической обоснованностью и высокой вычислительной эффективностью, что позволяет с достаточно высокой достоверностью обнаруживать скрытые медико-биологические закономерности при малом уровне исходной информации. Использование данной методики осуществлялось при помощи программных средств, разработанных в Институте вычислительного моделирования СО РАН г. Красноярска под руководством д.т.Н., проф. Лапко А.В. 
Программные модули обеспечивают возможность распознавания образов при ограниченном объеме обучающей выборки, оценивая вероятности ошибки распознавания образов, формирования наборов информативных признаков и оформления результатов классификации в многомерном пространстве признаков в виде последовательности таблиц, номограмм.

Результаты и обсуждение: Исходя из поставленной цели исследования у обследованных женщин были проанализированы факторы риска, которые могут обуславливать высокий риск развития осложненное течение АГ. Основываясь на данные проведенных ранее исследований [10, c. $66-74 ; 15$, с. 481$]$, в которых было установлено, что метаболические нарушения играют несомненную роль в становлении и прогрессировании АГ и часто предшествуют повышению АД, а пациенты, у которых гипертония сочетается с метаболическими расстройствами, представляют группу высокого риска развития осложнений, был определен набор признаков (факторов риска) из перечня показателей, обуславливающих риск развития прогнозируемого состояния (осложненное течение АГ) (табл. 1).

Таблииа 1.

Клиническая характеристика пациентов, включенных в исследование

\begin{tabular}{|l|c|c|c|}
\hline \multicolumn{1}{|c|}{ Показатель } & $\begin{array}{c}\text { Женщины с АГ } \\
(\mathrm{n}=54)\end{array}$ & $\begin{array}{c}\text { Женщины без АГ } \\
(\mathrm{n}=30)\end{array}$ & $p$ \\
\hline Возраст (лет) & $\begin{array}{c}46,91 \\
{[45,46-48,35]}\end{array}$ & $\begin{array}{c}42,50 \\
{[41,07-43,93]}\end{array}$ & $\mathrm{p}<0,001$ \\
\hline Уровни АД (мм рт.ст.) & & & \\
\hline САД & $\begin{array}{c}160,70 \\
{[155,96-165,45]}\end{array}$ & $\begin{array}{c}111,80 \\
{[106,40-117,20]}\end{array}$ & $\mathrm{p}<0,001$ \\
\hline ДАД & $\begin{array}{c}100,29 \\
{[97,64-102,95]}\end{array}$ & $\begin{array}{c}77,03 \\
{[74,13-79,94]}\end{array}$ & $\mathrm{p}<0,001$ \\
\hline чСС (уд. в мин. ) & $\begin{array}{c}73,50 \\
{[70,66-76,33]}\end{array}$ & $\begin{array}{c}71,63 \\
{[69,37-73,89]}\end{array}$ & $\mathrm{p}>0,05$ \\
\hline Гликемия (ммоль/л) & $\begin{array}{c}4,39 \\
{[4,07-4,73]}\end{array}$ & $\begin{array}{c}3,96 \\
{[3,58-4,33]}\end{array}$ & $\mathrm{p}>0,05$ \\
\hline натощак & $\begin{array}{c}6,93 \\
{[6,39-7,48]}\end{array}$ & $\begin{array}{c}5,52 \\
{[4,91-6,12]}\end{array}$ & $\mathrm{p}<0,001$ \\
\hline через 1 ч & $\begin{array}{c}5,15 \\
{[4,53-5,76]}\end{array}$ & $\begin{array}{c}4,27 \\
{[3,92-4,63]}\end{array}$ & $\mathrm{p}<0,05$ \\
\hline через 2 ч & & & \\
\hline Инсулин (мкед/мл) & & & \\
\hline
\end{tabular}


Окончание табл. 1.

\begin{tabular}{|c|c|c|c|}
\hline натощак & $\begin{array}{c}13,52 \\
{[8,73-18,31]}\end{array}$ & $\begin{array}{c}6,40 \\
{[0,17-12,63]}\end{array}$ & $\mathrm{p}<0,05$ \\
\hline через 1 ч & $\begin{array}{c}66,2808 \\
{[53,19-79,37]}\end{array}$ & $\begin{array}{c}34,26 \\
{[12,56-55,95]}\end{array}$ & $\mathrm{p}<0,05$ \\
\hline через 2 ч & $\begin{array}{c}38,87 \\
{[24,89-52,85]}\end{array}$ & $\begin{array}{c}13,06 \\
{[4,15-21,96]}\end{array}$ & $\mathrm{p}>0,05$ \\
\hline $\begin{array}{l}\text { Общий холестерин } \\
\text { (ммоль/л) }\end{array}$ & $\begin{array}{c}5,57 \\
{[5,09-6,05]} \\
\end{array}$ & $\begin{array}{c}4,72 \\
{[4,50-4,93]}\end{array}$ & $\mathrm{p}<0,05$ \\
\hline Индекс массы тела (кг/м²) & $\begin{array}{c}31,37 \\
{[29,66-33,07]}\end{array}$ & $\begin{array}{c}25,70 \\
{[24,10-27,31]}\end{array}$ & $\mathrm{p}<0,001$ \\
\hline \multicolumn{4}{|l|}{ Индекс массы тела (\%) } \\
\hline 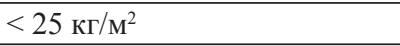 & 11,1 & 40 & $\mathrm{p}<0,05$ \\
\hline$\geq 25 \kappa \Gamma / \mathrm{M}^{2} \leq 29 \mathrm{\kappa} / \mathrm{M}^{2}$ & 33,3 & 43,3 & $\mathrm{p}<0,05$ \\
\hline 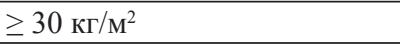 & 55,6 & 16,7 & $\mathrm{p}<0,001$ \\
\hline \multicolumn{4}{|l|}{ Употребления алкоголя (\%) } \\
\hline Не употребляют & 22,2 & 13,3 & $\mathrm{p}>0,05$ \\
\hline 1 раз в месяц и менее & 25,9 & 86,7 & $\mathrm{p}>0,05$ \\
\hline 1 раз в неделю и более & 1,9 & 0 & $\mathrm{p}>0,05$ \\
\hline \multicolumn{4}{|l|}{ Курение (\%) } \\
\hline Не курит & 98,2 & 100 & $\mathrm{p}>0,05$ \\
\hline Курит & 1,8 & 0 & $\mathrm{p}>0,05$ \\
\hline \multicolumn{4}{|l|}{ Наследственность } \\
\hline Отягощена & 76 & 46,7 & $\mathrm{p}<0,05$ \\
\hline Не отягощена & 24 & 53,3 & $\mathrm{p}<0,05$ \\
\hline
\end{tabular}

Далее, с применением методики распознавания образов проводился многомерный анализ взаимосвязи между исследуемыми состояниями $\mathrm{S}$ (S1-нормальное и $\mathrm{S} 2$-осложненное клиническое течение АГ) и выраженностью факторов риска СС3 осложняющих клиническое течение АГ. Математический анализ результатов обследования по построенной математической модели выявил различия между исследуемыми состояниями $\mathrm{S}$ и выраженностью показателей углеводного и липидного обменов таких ФР, как гиперинсулинемия, нарушенная толерантность к углеводам (НТУ), гиперхолестеринемия (ГХС), избыточная масса тела (ИзМТ).

Наиболее информативным, давшим наименьшую ошибку распознания образов, оказался следующий набор признаков: систолическое (САД) и диастолическое артериальное давление (ДАД), индекс массы тела (Кетле) (ИМТ), уровень общего холестерина сыворотки крови (ОХC), уро- 
вень глюкозы натощак и через 2 часа после нагрузки глюкозой в ходе ПГТТ. Данные, полученные в результате математического моделирования, представленные в виде прогнозных номограмм, позволили графически отобразить области распределения состояний низкого и высокого риска осложнённого течения АГ у женщин. Подобные показатели, с высокой информативностью, были определены ранее и при обследовании мужчин [25].

Из полученного набора прогнозных номограмм, в качестве примера представлены номограммы (рис.1-2), отражающие влияние на клиническое течение АГ уровней САД и ДАД, величин ИМТ, тощаковой и постпрандиальной гликемии, ОХС.

Анализ номограмм, отражающих влияние ОХC на прогноз развития осложненного течения АГ в зависимости от тощаковой глюкозы и ДАД, показал, что область низкого риска при уровне ДАД 90-99 мм рт.ст. располагалась среди значений ОХС $<4,5$ ммоль/л и уровня тощаковой глюкозы $<5,0$ ммоль/л Увеличение значений ДАД от 100 до 110 мм рт.ст. увеличивало область высокого риска и смещало границу между состояниями $\mathrm{S}$ в сторону значений тощаковой глюкозы $<5,25$ ммоль/л и значений $\mathrm{OXC}<5,25$ ммоль/л. Дальнейшее увеличение уровня ДАД от 110 мм рт.ст. и выше при значениях гликемии 5,5 ммоль/л приводит к расширению области высокого риска за счет уменьшения уровня ОХС до 5,5 ммоль/л (рис. 1).

Подобные закономерности были установлено и при влиянии ОХС на прогноз развития осложненного течения АГ в зависимости от постпрандиальной гликемии у женщин с АГ при различных значениях ДАД. Установлено, что область низкого риска располагалась среди значений ОХС < 5 ммоль/л и глюкозы $<5,5$ ммоль/л при уровне ДАД 90-99 мм рт.ст. При дальнейшем увеличение ДАД от 100 до 110 мм рт.ст. величина неблагоприятных значений гликемии и ОХС не менялась. Однако при значениях ДАД выше 110 мм рт.ст. расширялась область высокого риска за счет уменьшения значений ОХС до 4,5 ммоль/л, при тех же значениях постпрандиальной гликемии.

Анализ номограмм, отражающих влияние тощаковой глюкозы в зависимости от ИМТ на прогноз развития осложненного течения АГ при различных значениях ДАД, показал, что область низкого риска располагалась среди значений глюкозы натощак $<5,5$ ммоль/л и ИМТ $<25,0$ кг/ $\mathrm{M}^{2}$ при уровне ДАД 90-99 мм рт.ст. При увеличении ДАД в пределах от 100 до 110 мм рт.ст. увеличивалась область высокого риска, смещающая 
границу между состояниями $\mathrm{S}$ в сторону более низких значений глюкозы натощак (5,0 ммоль/л), при этом величина неблагоприятных значений ИМТ не изменялась. Последующее увеличение ДАД от 110 до 120 мм рт.ст. смещало границу в сторону более низких значений глюкозы $(4,5$ ммоль/л) не меняя величину ИМТ. При увеличении значений ДАД выше 120 мм рт.ст. отмечается смещение границы в сторону более низких показателей ИМТ до 23,0 кг/м² , при этом величина неблагоприятных значений глюкозы натощак не менялась.

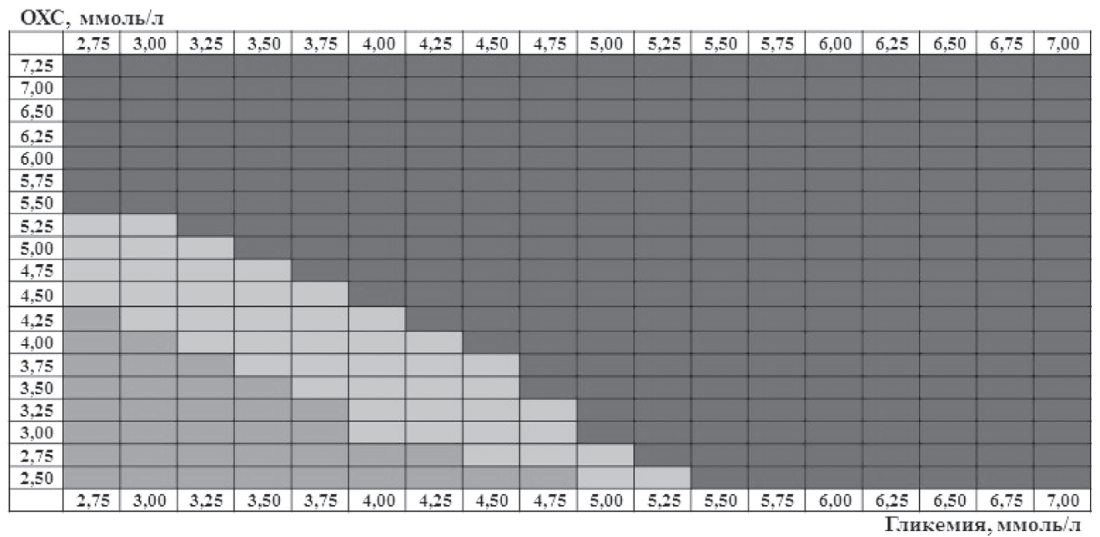

Рис. 1. Прогнозная номограмма тяжести течения АГ у женшин в зависимости от уровней АД, общего холестерина сыворотки крови и тощаковой гликемии

Область низкого риска

Область высокого риска неблагоприятного течения при ДАД более 90 - 99 мм рт.ст.

Область высокого риска неблагоприятного течения при ДАД более $100-109$ мм рт.ст.

Проанализировано влияние постпрандиальной гликемии при различных значениях ДАД на прогноз в зависимости от ИМТ (рис. 2). При уровнях постпрандиальной гликемии $<6$ ммоль/л область низкого риска при ДАД менее 100 мм рт.ст. располагалась в области значений ИМТ <

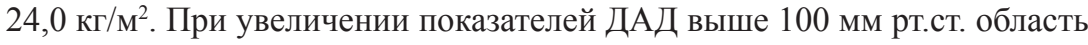
высокого риска смещалась в сторону более низких значений постпрандиальной гликемии от 7 до 5,75 ммоль/л, не изменяя величины неблагоприятных значений ИМТ.

Также установлено, что при повышении уровня глюкозы натощак до 5,7 ммоль/л у женщин неблагоприятным показателем индекса массы тела

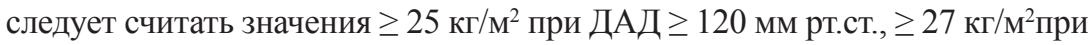

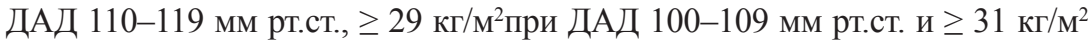


для ДАД 90-99 мм рт.ст. соответственно. Идентичным показателям неблагоприятного порога значений ИМТ, при ДАД 100-109, 110-119, $\geq 120$ мм рт.ст., соответствовали уровни постпрандиальной гликемии - 7,3 ммоль/л, а при значениях ДАД 90-99 мм рт.ст. - 7,5 ммоль/л соответственно.

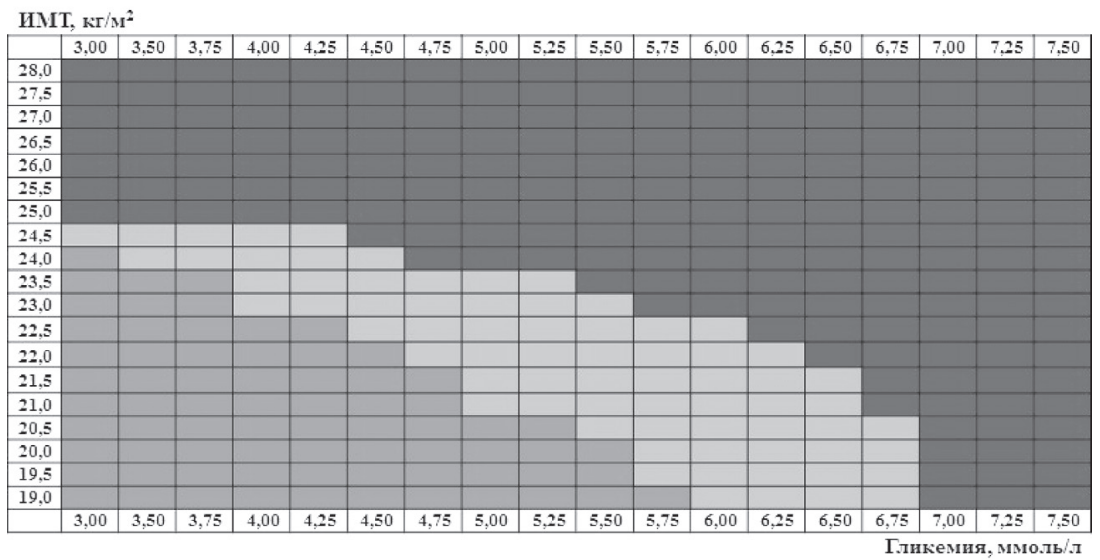

Рис. 2. Прогнозная номограмма тяжести течения АГ у женщин в зависимости от уровней АД, индекса массы тела и постпрандиальной гликемии.

Область низкого риска

Область высокого риска неблагоприятного течения при ДАД более 90 - 99 мм рт.ст.

Область высокого риска неблагоприятного течения при ДАД более $100-109$ мм рт.ст.

Подобная закономерность отмечались и при обследовании мужчин [ 25 , эл.ресурс], однако пороговые значения ИМТ были ниже, чем у женщин. Также, как и у женщин, у мужчин при указанных цифрах ИМТ и АД неблагоприятное течение заболевания наблюдается при повышении ОХC, причем чем выше уровень ДАД, тем ниже показатели ОХС, оказывающие неблагоприятное воздействие на прогноз заболевания [25, эл.ресурс].

Заключение. Таким образом в ходе проведенного исследования установлено, что у женщин на клиническое течение АГ влияет повышение уровня АД, значений ИРИ, тощаковой и постпрандиальной глюкозы, ОХС, ИМТ, то есть показатели, входящие в симтомокомплекс метаболического синдрома. Сформированный по результатам математического моделирования, индивидуальный набор признаков (факторов риска) из перечня показателей, обуславливающих риск развития прогнозируемого состояния 
(осложненное течение АГ), позволил построить прогнозные номограммы, на которых выделены области низкого, среднего и высокого риска развития осложненного течения АГ, что не только позволит индивидуально (персонифицированно) рассчитать для каждого пациента степень риска , но и определить параметры необходимого изменения уровня управляемых факторов риска, определяющих нахождение в области высокого риска.

\section{Список литературы}

1. Артамонова Г.В., Максимов С.А., Индукаева Е.В. и др. Прогнозирование артериальной гипертензии у женщин в зависимости от возраста и характера трудовой деятельности // Бюллетень сибирской медицины. 2011. T.10. № 4. C. 141-145.

2. Ахминеева А.Х., Полунина О.С., Севостьянова И.В. и др. Прогнозирование развития артериальной гипертензии и ишемической болезни сердца у больных хронической обструктивной болезнью легких // Курский научно-практический вестник «Человек и его здоровье». 2014. № 4. С. 29-34.

3. Гоголашвили Н.Г., Савченко А.А., Яскевич Р.А. Метод оценки ухудшения течения желудочковой экстрасистолии у больных с постинфарктным кардиосклерозом: медицинская технология Красноярск, 2016. 19 с.

4. Грицинская В.Л., Москаленко О.Л. Использование компьютерных технологий при проведении диспансеризации детского населения республики Тыва // В мире научных открытий. 2017. Т. 9. № 2. С. 158-167.

5. Деревянных Е.В., Яскевич Р.А. Изучение и сравнительный анализ показателей центральной гемодинамики и артериального давления у пожилых мигрантов Крайнего Севера с артериальной гипертензией // Клиническая геронтология. 2017. Т. 23, № 9-10. С. 23-25.

6. Лапко А.В., Лапко В.А. Непараметрические системы обработки информации и принятия решений. Красноярск: СибГАУ, 2014. 382 с.

7. Лапко А.В., Лапко В.А. Непараметрические системы обработки неоднородной информации. Новосибирск: Наука, 2007. 174 с.

8. Лапко А.В., Поликарпов Л.С., Манчук В.Т. Автоматизация научных исследований в медицине. Новосибирск: Наука, 1996. 270 с.

9. Москаленко О.Л. Влияние городского техногенного загрязнения на морфофункциональное состояние юношей: автореф. дисс. канд. биол. наук. Красноярск, 2014. 18 с.

10. Никитин Ю.П., Воевода М.И., Симонова Г.И. Сахарный диабет и метаболический синдром в Сибири и на Дальнем Востоке // Вестник РАМН. 2012. №1. С. 66-74. 
11. Оленская Т.Л., Коневалова Н.Ю., Губарев Ю.Д., Бирюкова И.В. Прогнозирование развития нефатальных исходов у пациентов с артериальной гипертензией старших возрастных групп в концепции гериатрических синдромов // Современные проблемы науки и образования. 2015. № 1-1. С. 1383.

12. Поликарпов Л.С., Лапко А.В., Хамнагадаев И.И., Яскевич Р.А. Метеотропные реакции сердечно-сосудистой системы и их профилактика. Новосибирск: Наука, 2005. 195 с.

13. Поликарпов Л.С., Хамнагадаев И.И., Иванова Е.Б. и др. Частота сердечно-сосудистой патологии, содержание микроэлементов в различных средах в условиях Севера // Сибирский медицинский журнал (г. Томск). 2005. T. 20, № 2. C. 55-57.

14. Поликарпов Л.С., Хамнагадаев И.И., Яскевич Р.А. и др. Ишемическая болезнь сердца (распространенность, профилактика, адаптация и реадаптация в различных экологических условиях). Красноярск: Изд-во КрасГМУ, $2011.328 \mathrm{c}$.

15. Поликарпов Л.С., Яскевич Р.А., Хамнагадаев И.И. и др. Влияние компонентов метаболического синдрома на клиническое течение ишемической болезни сердца у мужчин // Современные проблемы науки и образования. 2014. № 5. C. 481.

16. Пуликов А.С., Москаленко О.Л. Особенности экологической морфологии юношей Сибири в условиях городского антропотехногенного загрязнения // В мире научных открытий. 2015. № 6.1 (66). С. 393-407.

17. Савченко А.А. Способ прогнозирования геморрагических осложнений после химиотерапии у больных острыми лейкозами / А.А. Савченко, O.В. Смирнова, В.Т. Манчук, В.И. Москов. Патент на изобретение RUS 2324190, 15.02.2006.

18. Савченко А.А. Способ прогнозирования инфекционных осложнений после химиотерапии у больных острыми лейкозами / А.А. Савченко, О.В. Смирнова, B.T. Манчук, В.И. Москов. Патент на изобретение RUS 2315305, 08.11.2005.

19. Суспицына И.Н., Сукманова И.А. Факторы риска и прогнозирование развития инфаркта миокарда у мужчин различных возрастных групп // Российский кардиологический журнал. 2016. № 8 (136). С. 58-63.

20. Хамнагадаев И.И., Яскевич Р.А., Поликарпов Л.С., Новгородцева Н.Я. Распространенность артериальной гипертонии и избыточной массы тела среди сельского населения северных регионов // Сибирский медицинский журнал (г. Томск). 2004. Т. 19, № 4. С. 94-96.

21. Филимонова Л.А., Яскевич Р.А., Давыдов Е.Л. Вопросы формирования и течения артериальной гипертонии в пожилом и старческом возрасте // 
Современные проблемы науки и образования. 2016. № 6. http://scienceeducation.ru/ru/article/view?id $=25458$

22. Яскевич Р.А., Деревянных Е.В., Балашова Н.А. Использование показателей соматотипирования у мужчин в построении математических моделей прогноза развития артериальной гипертонии // Международный журнал прикладных и фундаментальных исследований. 2015. № 1-1. С. 64-69.

23. Яскевич Р.А., Деревянных Е.В., Балашова Н.А., Козлов Е.В. Использование показателей соматометрии у женщин в построении математической модели прогноза развития артериальной гипертонии // Современные проблемы науки и образования. 2015. № 6-0. С. 188.

24. Яскевич Р.А. Избыточная масса тела и особенности компонентного состава массы тела у пожилых мигрантов Крайнего Севера // Клиническая геронтология. 2017. Т. 23, № 9-10. С. 79-81.

25. Яскевич Р.А. Применение методов математического моделирования в прогнозе тяжести клинического течения артериальной гипертонии у мужчин // Современные проблемы науки и образования. 2016. № 6. URL: https:// www.science-education.ru/ru/article/view?id $=25563$

26. Яскевич Р.А., Хамнагадаев И.И., Деревянных Е.В. и др. Тревожно-депрессивные расстройства у пожилых мигрантов Крайнего Севера в период реадаптации к новым климатическим условиям // Успехи геронтологии. 2014. T. 27, № 4. C. 672-677.

27. Artyukhov I.P., Grinshtein Y.I., Petrova M.M. et al. Prevalence of arterial hypertension in the Krasnoyarsk Krai (Siberia, Russia). BMC Cardiovascular Disorders. 2017. 17(1):138. doi: 10.1186/s12872-017-0559-5.

28. Graham I., Atar D., Borch-Johnsen K. et al. European guidelines on cardiovascular disease prevention in clinical practice: full text. Fourth Joint Task Force of the European Society of Cardiology and other societies on cardiovascular disease prevention in clinical practice (constituted by representatives of nine societies and by invited experts). Eur. J. Cardiovasc. Prev. Rehabil. 2007. Vol. 14. Suppl. 2, pp. 1-113.

29. Yaskevich R.A., Khamnagadaev I.I., Dereviannikh Ye.V. et al. Anxiety depressive disorders in elderly migrants of the far North in the period of readaptation to new climatic conditions. Advances in Gerontology. 2015. T. 5. № 3. C. 157-162.

\section{References}

1. Artamonova G.V., Maksimov S.A., Indukaeva E.V. i dr. Prognozirovanie arterial'noy gipertenzii u zhenshchin $\mathrm{v}$ zavisimosti ot vozrasta i kharaktera trudovoy deyatel'nosti [Forecasting of arterial hypertension in women depending on 
the age and type of labor activity]. Byulleten' sibirskoy meditsiny [Bulletin of Siberian Medicine]. 2011. V.10. № 4, pp. 141-145.

2. Akhmineeva A.Kh., Polunina O.S., Sevost'yanova I.V. et al. Prognozirovanie razvitiya arterial'noy gipertenzii i ishemicheskoy bolezni serdtsa u bol'nykh khronicheskoy obstruktivnoy bolezn'yu legkikh [Forecasting the development of arterial hypertension and coronary heart disease in patients with chronic obstructive pulmonary disease]. Kurskiy nauchno-prakticheskiy vestnik "Chelovek i ego zdorov'e" [Kursk Scientific and Practical Herald "The Man and His Health"]. 2014. № 4, pp. 29-34.

3. Gogolashvili N.G., Savchenko A.A., Yaskevich R.A. Metod otsenki ukhudsheniya techeniya zheludochkovoy ekstrasistolii u bol'nykh s postinfarktnym kardiosklerozom: meditsinskaya tekhnologiya [Method for assessing the deterioration of ventricular extrasystole in patients with postinfarction cardiosclerosis: medical technology]. Krasnoyarsk. 2016. 19 p.

4. Gritsinskaya V.L., Moskalenko O.L. Ispol'zovanie komp'yuternykh tekhnologiy pri provedenii dispanserizatsii detskogo naseleniya respubliki Tyva [Use of computer technologies in the course of clinical examination of the children of the Republic of Tyva]. V mire nauchnykh otkrytiy [Siberian Journal of Life Sciences and Agriculture]. 2017. V. 9. № 2, pp. 158-167.

5. Derevyannykh E.V., Yaskevich R.A. Izuchenie i sravnitel'nyy analiz pokazateley tsentral'noy gemodinamiki i arterial'nogo davleniya u pozhilykh migrantov Kraynego Severa s arterial'noy gipertenziey [Study and comparative analysis of central hemodynamics and arterial pressure in elderly migrants of the Far North with arterial hypertension]. Klinicheskaya gerontologiya [Clinical gerontology]. 2017. V. 23. № 9-10, pp. 23-25.

6. Lapko A.V., Lapko V.A. Neparametricheskie sistemy obrabotki informatsii $i$ prinyatiya resheniy [Nonparametric systems for information processing and decision making]. Krasnoyarsk: SibGAU. 2014. 382 p.

7. Lapko A.V., Lapko V.A. Neparametricheskie sistemy obrabotki neodnorodnoy informatsii [Nonparametric systems for processing heterogeneous information]. Novosibirsk: Nauka. 2007. 174 p.

8. Lapko A.V., Polikarpov L.S., Manchuk V.T. Avtomatizatsiya nauchnykh issledovaniy $v$ meditsine [Automation of scientific research in medicine]. Novosibirsk: Nauka. 1996. 270 p.

9. Moskalenko O.L. Vliyanie gorodskogo tekhnogennogo zagryazneniya na morfofunktsional'noe sostoyanie yunoshey [Influence of urban man-made pollution on the morphofunctional state of young men]. Krasnoyarsk, 2014. 18 p.

10. Nikitin Yu.P., Voevoda M.I., Simonova G.I. Sakharnyy diabet i metabolicheskiy sindrom v Sibiri i na Dal'nem Vostoke [Diabetes mellitus and metabolic 
syndrome in Siberia and the Far East]. Vestnik RAMN [Bulletin of the Russian Academy of Medical Sciences]. 2012. №1, pp. 66-74.

11. Olenskaya T.L., Konevalova N.Yu., Gubarev Yu.D., Biryukova I.V. Prognozirovanie razvitiya nefatal'nykh iskhodov u patsientov $\mathrm{s}$ arterial'noy gipertenziey starshikh vozrastnykh grupp v kontseptsii geriatricheskikh sindromov [Predicting the development of non-fatal outcomes in patients with arterial hypertension of older age groups in the concept of geriatric syndromes]. Sovremennye problemy nauki i obrazovaniya [Modern problems of science and education]. 2015. № 1-1. P. 1383.

12. Polikarpov L.S., Lapko A.V., Khamnagadaev I.I., Yaskevich R.A. Meteotropnye reaktsii serdechno-sosudistoy sistemy i ikh profilaktika [Meteotropic reactions of the cardiovascular system and their prevention]. Novosibirsk: Nauka. 2005. $195 \mathrm{p}$.

13. Polikarpov L.S., Khamnagadaev I.I., Ivanova E.B. et al. Chastota serdechno-sosudistoy patologii, soderzhanie mikroelementov $\mathrm{v}$ razlichnykh sredakh $\mathrm{v}$ usloviyakh Severa [The frequency of cardiovascular pathology, the content of trace elements in various environments in the North]. Sibirskiy meditsinskiy zhurnal (Tomsk) [Siberian Medical Journal (Tomsk)]. 2005. V. 20. № 2, pp. 55-57.

14. Polikarpov L.S., Khamnagadaev I.I., Yaskevich R.A. et al. Ishemicheskaya bolezn'serdtsa (rasprostranennost', profilaktika, adaptatsiya i readaptatsiya $v$ razlichnykh ekologicheskikh usloviyakh) [Coronary heart disease (prevalence, prevention, adaptation and re-adaptation in various environmental conditions)]. Krasnoyarsk: Izd-vo KrasGMU. 2011. 328 p.

15. Polikarpov L.S., Yaskevich R.A., Khamnagadaev I.I. et al. Vliyanie komponentov metabolicheskogo sindroma na klinicheskoe techenie ishemicheskoy bolezni serdtsa u muzhchin [Influence of metabolic syndrome components on clinical course of coronary heart disease in men]. Sovremennye problemy nauki i obrazovaniya [Modern problems of science and education]. 2014. № 5. S. 481.

16. Pulikov A.S., Moskalenko O.L. Osobennosti ekologicheskoy morfologii yunoshey Sibiri v usloviyakh gorodskogo antropotekhnogennogo zagryazneniya [Peculiarities of the ecological morphology of the young men of Siberia in the conditions of urban anthropogenic contamination]. V mire nauchnykh otkrytiy [Siberian Journal of Life Sciences and Agriculture]. 2015. № 6.1 (66), pp. 393-407.

17. Savchenko A.A., Smirnova O.V., Manchuk V.T., Moskov V.I. Sposob prognozirovaniya gemorragicheskikh oslozhneniy posle khimioterapii u bol'nykh ostrymi leykozami [A method for predicting hemorrhagic complications after chemotherapy in patients with acute leukemia]. Patent na izobretenie RUS 2324190, 15.02.2006. 
18. Savchenko A.A., Smirnova O.V., Manchuk V.T., Moskov V.I. Sposob prognozirovaniya infektsionnykh oslozhneniy posle khimioterapii u bol'nykh ostrymi leykozami [The method of predicting infectious complications after chemotherapy in patients with acute leukemia]. Patent na izobretenie RUS 2315305, 08.11.2005.

19. Suspitsyna I.N., Sukmanova I.A. Faktory riska i prognozirovanie razvitiya infarkta miokarda u muzhchin razlichnykh vozrastnykh grupp [Risk factors and prognosis of the development of myocardial infarction in men of different age groups]. Rossiyskiy kardiologicheskiy zhurnal [Russian Cardiology Journal]. 2016. № 8 (136), pp. 58-63.

20. Khamnagadaev I.I., Yaskevich R.A., Polikarpov L.S., Novgorodtseva N.Ya. Rasprostranennost' arterial'noy gipertonii i izbytochnoy massy tela sredi sel'skogo naseleniya severnykh regionov [The prevalence of arterial hypertension and overweight among rural population of northern regions]. Sibirskiy meditsinskiy zhurnal (Tomsk) [Siberian Medical Journal (Tomsk)]. 2004. V. 19. № 4, pp. 94-96.

21. Filimonova L.A., Yaskevich R.A., Davydov E.L. Voprosy formirovaniya i techeniya arterial'noy gipertonii v pozhilom i starcheskom vozraste [Questions of formation and course of arterial hypertension in elderly and senile age]. Sovremennye problemy nauki i obrazovaniya [Modern problems of science and education]. 2016. № 6. http://science-education.ru/ru/article/view?id=25458

22. Yaskevich R.A., Derevyannykh E.V., Balashova N.A. Ispol'zovanie pokazateley somatotipirovaniya $\mathrm{u}$ muzhchin $\mathrm{v}$ postroenii matematicheskikh modeley prognoza razvitiya arterial'noy gipertonii [Use of somatotyping indices in men in the construction of mathematical models for the prediction of the development of arterial hypertension]. Mezhdunarodnyy zhurnal prikladnykh i fundamental'nykh issledovaniy [International Journal of Applied and Fundamental Research]. 2015. № 1-1, pp. 64-69.

23. Yaskevich R.A., Derevyannykh E.V., Balashova N.A., Kozlov E.V. Ispol'zovanie pokazateley somatometrii $\mathrm{u}$ zhenshchin $\mathrm{v}$ postroenii matematicheskoy modeli prognoza razvitiya arterial'noy gipertonii [The use of somatometry indicators in women in the construction of a mathematical model for the prediction of the development of arterial hypertension]. Sovremennye problemy nauki i obrazovaniya [Modern problems of science and education]. 2015. № 6-0. P. 188.

24. Yaskevich R.A. Izbytochnaya massa tela i osobennosti komponentnogo sostava massy tela u pozhilykh migrantov Kraynego Severa [Overweight and features of the component composition of body weight in elderly migrants of the Far North]. Klinicheskaya gerontologiya [Clinical gerontology]. 2017. V. 23. № 9-10, pp. 79-81. 
25. Yaskevich R.A. Primenenie metodov matematicheskogo modelirovaniya $v$ prognoze tyazhesti klinicheskogo techeniya arterial'noy gipertonii u muzhchin [Application of methods of mathematical modeling in the forecast of severity of the clinical course of arterial hypertension in men]. Sovremennye problemy nauki i obrazovaniya [Modern problems of science and education]. 2016. № 6. https://www.science-education.ru/ru/article/view?id=25563

26. Yaskevich R.A., Khamnagadaev I.I., Derevyannykh E.V. et al. Trevozhno-depressivnye rasstroystva u pozhilykh migrantov Kraynego Severa v period readaptatsii k novym klimaticheskim usloviyam [Anxiety-depressive disorders in elderly migrants of the Far North in the period of readaptation to new climatic conditions]. Uspekhi gerontologii [Successes of gerontology]. 2014. V. 27. № 4, pp. 672-677.

27. Artyukhov I.P., Grinshtein Y.I., Petrova M.M. et al. Prevalence of arterial hypertension in the Krasnoyarsk Krai (Siberia, Russia). BMC Cardiovascular Disorders. 2017. 17(1):138. doi: 10.1186/s12872-017-0559-5.

28. Graham I., Atar D., Borch-Johnsen K. et al. European guidelines on cardiovascular disease prevention in clinical practice: full text. Fourth Joint Task Force of the European Society of Cardiology and other societies on cardiovascular disease prevention in clinical practice (constituted by representatives of nine societies and by invited experts). Eur. J. Cardiovasc. Prev. Rehabil. 2007. Vol. 14. Suppl. 2, pp. 1-113.

29. Yaskevich R.A., Khamnagadaev I.I., Dereviannikh Ye.V. et al. Anxiety depressive disorders in elderly migrants of the far North in the period of readaptation to new climatic conditions. Advances in Gerontology. 2015. T. 5. №3. S. $157-162$.

\section{ДАННЫЕ ОБ АВТОРАХ}

Яскевич Роман Анатольевич, доцент кафедры пропедевтики внутренних болезней и терапии, ведущий научный сотрудник группы патологии сердечно-сосудистой системы, кандидат медицинских наук, доцент

Федеральное государственное бюджетное научное учреждение «Научно-исследовательский институт медичинских проблем Севера»; ГБОУ ВПО «КрасГМУ им. проф. Ф.В. Войно-Ясенеикого» МЗ РФ ул. Партизана Железняка, 32, г. Красноярск, 660022, Российская Федерация; ул. Партизана Железняка, 1а, г. Красноярск, 660022, Российская Федерация cardio@impn.ru 
Москаленко Ольга Леонидовна, старший научный сотрудник, кандидат биологических наук

Федеральное государственное бюджетное научное учреждение «Научно-исследовательский институт медицинских проблем Севера» ул. Партизана Железняка, 32, г. Красноярск, 660022, Российская Федераиия

gre-ll@mail.ru

\section{DATA ABOUT THE AUTHORS}

Yaskevich Roman Anatolyevich, Associate Professor at Department of Propedeutics of Internal Diseases and Therapy, Leading Researcher of the Group Pathology of the Cardiovascular System, Candidate of Medical Science, Docent

Scientific Research Institute of medical problems of the North; Krasnoyarsk State Medical University named after Professor V.F. Voino-Yasenetzkiy

1a, P. Zheleznyaka Str., Krasnoyarsk, 660022, Russian Federation; 3g, P. Zheleznyaka Str., Krasnoyarsk, 660022, Russian Federation cardio@impn.ru

Moskalenko Olga Leonidovna, Senior Researcher, Candidate of Biological Sciences

Scientific Research Institute of medical problems of the North 3g, P. Zheleznyaka Str., Krasnoyarsk, 660022, Russian Federation gre-ll@mail.ru 